\title{
Essais
}

ESSAIS

Revue interdisciplinaire d'Humanités

Hors-série 6 | 2021

Agrobiodiversité et territoires

\section{La parole citoyenne face aux enjeux agroalimentaires et environnementaux : étude sémiotique des États généraux de l'alimentation}

Citizens' voices on agri-food and environmental issues: a semiotic study of the National Assembly on Food

\section{Céline Cholet}

\section{CpenEdition}

\section{Journals}

Édition électronique

URL : https://journals.openedition.org/essais/7403

DOI : 10.4000/essais.7403

ISSN : 2276-0970

Éditeur

École doctorale Montaigne Humanités

Édition imprimée

Date de publication : 1 mars 2021

Pagination : 65-77

ISBN : 978-2-492780-00-4

ISSN : 2417-4211

Référence électronique

Céline Cholet, « La parole citoyenne face aux enjeux agroalimentaires et environnementaux : étude sémiotique des États généraux de l'alimentation », Essais [En ligne], Hors-série 6 | 2021, mis en ligne le 16 mars 2021, consulté le 18 janvier 2023. URL : http://journals.openedition.org/essais/7403 ; DOI https://doi.org/10.4000/essais.7403 


\section{La parole citoyenne face aux enjeux agroalimentaires et environnemen- taux : étude sémiotique des États généraux de l'alimentation}

Céline Cholet

Depuis les années 2000, les enjeux sanitaires et environnementaux, ainsi que les préoccupations liées au changement climatique occupent une place importante dans la gestion de la politique agricole (à l'échelle française comme européenne $)^{1}$. Ce constat nous conduit à nous intéresser aux États généraux de l'alimentation et particulièrement à la plateforme participative de la consultation citoyenne (ouverte du 20 juillet au 10 novembre 2017)2 ${ }^{2}$. Cette dernière nous paraît être un espace privilégié pour aborder le point de vue de la société civile, ses attentes, ses préoccupations, mais aussi, sa représentation du monde agricole. C'est pourquoi nous en avons fait notre objet d'étude dans le cadre du séminaire "La biodiversité agroalimentaire et horticole au cœur de projets de territoires : recherches en cours et retours d'expériences ", dont cet article en est le prolongement ${ }^{3}$.

Dans ce qui suit, nous étudierons seulement la consultation menée sur la transformation agricole française, l'une des dix proposées par les États généraux de l'alimentation. Le cadre de l'étude ne pouvant envisager toutes les consultations, nous nous sommes concentrés sur celle qui a recueilli le plus fort taux de participation. Elle se situe devant les consultations sur la rémunération des producteurs, les modes de consommation et l'information des consommateurs. Cette étude se présente comme un préalable pour interroger la question de la production alimentaire, mais aussi, et peut-être surtout, pour pointer les valeurs en jeu.

1 Henri Nallet, Philippe Tillous-Borde, " Pour une nouvelle politique agricole ", Les nouveaux équilibres agroalimentaires mondiaux, Paris, Presses Universitaires de France, 2011, p. 119-125.

2 Site de la consultation citoyenne : egalimentation.gouv.fr.

3 Séminaire inter-doctoral, École Doctorale 480 Montaigne-Humanités, « La biodiversité agroalimentaire et horticole au cœur de projets de territoires : recherches en cours et retours d'expériences ", Université Bordeaux Montaigne, 14 et 15 mai 2019. 
Si le principe d'une plateforme participative est assez récent dans les pratiques politiques ${ }^{4}$, notons, en revanche, que le débat sur les modes de productions alimentaires n'est pas nouveau. Marquées par les crises sanitaires, les années 1990 et 2000 ont poussé les citoyen(ne)s à s'interroger sur leur consommation et la manière dont ces biens sont produits 5 . Mais depuis peu, il nous semble que ce sont davantage les problématiques du réchauffement climatique et de la biodiversité qui sont le moteur d'un renouvellement du regard citoyen sur les pratiques agricoles. En effet, les dérèglements climatiques et la disparition accélérée de la biodiversité (faune et flore confondues) s'imposent de plus en plus dans l'actualité quotidienne. Ils invitent le/la citoyen(ne) à ce (re)mobiliser. Cette préoccupation est d'autant plus grande que la médiatisation des recherches scientifiques et leurs publications alimentent largement les débats (le rapport de l'IPBES ou les rapports du GIEC ${ }^{6}$ de 2014 et 2018 par exemple).

Ainsi, si les années 1990 et 2000 sont marquées par les crises sanitaires successives telles que la vache folle, la grippe aviaire ou porcine, et ont poussé les citoyen(ne)s à plus de vigilance (par la modification de leur mode de consommation, entre autres), qu'en est-il aujourd'hui à partir de l'angle d'une crise majeure du climat et de la biodiversité mondiale?

En prenant appui sur la consultation citoyenne, nous nous interrogerons sur les problématiques suivantes : quelles propositions le panel de citoyen(ne)s retient-il pour répondre à la question " Comment accompagner la transformation de notre agriculture " ? Le climat et la préservation de la biodiversité sont-ils présents, si oui, comment ? Le modèle agricole dominant (l'agriculture dite "conventionnelle") est-il remis en cause ? Si oui, comment ? En effet, malgré les crises sanitaires précédentes qui ont pointé et revu la sécurité des aliments (par une traçabilité renforcée notamment), elles n'ont pas pour autant renversé ce modèle hérité des années 1960-1970. Au final, quelle(s) représentation(s) des modèles agricoles les investissements sémantiques que nous relèverons suggèrent-ils ? Pour y répondre, nous mettons au cœur de notre réflexion les propositions les plus votées favorablement lors de la consultation étudiée, considérant qu’elles sont des actes langagiers porteurs de valeurs. Nous pensons en effet que ces manifestations discursives reflètent ce qui affecte les citoyen(ne)s, et donc, ce qu'ils retiennent comme pertinent pour opérer une transformation.

4 Pensons également à la plateforme du Grand Débat national lancé le 10 décembre 2018.

5 Estelle Masson et al., "La crise de la vache folle : "psychose", contestation, mémoire et amnésie ", Connexions, vol. n 80, 2003/2, p. 93-104 ; Estelle Masson, "Représentations de l'alimentation : crise de la confiance et crises alimentaires ", Bulletin de psychologie, vol. 514, n 4, 2011, p. 307-314.

6 Site The Intergovernmental Panel on Climate Change : https://www.ipcc.ch/. 
À partir d'une approche sémiotique, nous tenterons d'apporter des réponses à nos interrogations, puis nous les mettrons en perspective. Pour commencer, nous expliciterons les choix opérés, ensuite la méthode et les notions majeures qui s'inscrivent au cœur de notre réflexion. Puis, nous procéderons à l'observation du corpus et à sa discussion.

\section{La transformation de l'agriculture française}

Les propositions de la consultation : "Comment accompagner la transformation de notre agriculture?»

Au total, les États généraux de l'alimentation de 2017 ont rassemblé 4096 contributions (propositions + commentaires), 42724 votes, et 10475 participants. Pour la consultation qui nous intéresse ici, à savoir "Comment accompagner la transformation de notre agriculture ?", on compte 230 propositions. Dans le cadre de cet article, nous avons constitué un corpus d'étude (au sens défini par François Rastier ${ }^{7}$ ) d'une cinquantaine d'entre elles. Comme évoqué plus haut, nous avons choisi de réunir les propositions qui ont reçu le plus grand nombre de votes favorables, en d'autres termes, celles qui semblent davantage avoir fait l'objet d'un consensus. Parmi elles, remarquons que les quatre premières ont été formulées par les États généraux de l'alimentation. Les dix suivantes ${ }^{8}$ relèvent d'organisations à but non lucratif ou lucratif, comme l'Association végétarienne de France, le Syndicat Réseau Entreprises Bio Agroalimentaire, Synabio, l'Association de défense de l'environnement agréée par le ministère de l'Écologie, Générations Futures, et le réseau de distribution Biocoop. À leur suite, on compte un ensemble de propositions issu de profils de citoyen(ne)s et d'autres organisations. Constatons ainsi la présence non négligeable de ces dernières, leur savoir-faire leur confère une légitimité.

Passons à la méthodologie sur laquelle s’appuie cette réflexion.

\section{L'approche sémiotique : modèle conventionnel versus modèles alternatifs}

Au cœur de nos propos, il y a la notion d'acte langagier. Il s'agit d'aborder le corpus comme un ensemble de manifestations discursives pertinent pour interroger la construction des représentations de leurs énonciateurs, et des

7 Le corpus d'étude est délimité par les besoins de l'application. Il se différencie de l'archive, du corpus de référence, ou encore du sous-corpus de travail. François Rastier, La mesure et le grain : sémantique de corpus, Paris, Honoré Champion éditeur, 2011, p. 36.

8 L'identification des quatre puis des dix premières propositions a été réalisée à partir du décompte du nombre de votes favorables aux propositions «Comment accompagner la transformation de notre agriculture? ". 
processus de valorisation qui leur sont associés, partagés de façon plus ou moins étendue avec une communauté. En effet, malgré un objectif unanime de soutien des modes de production durables, sur différentes échelles, cette consultation met en évidence différents moyens d'y parvenir, entre " état de choses " et "états d'âme ", valorisant des modèles agricoles contraires ou contradictoires. En tant qu'investissements sémantiques, ces énoncés permettent d'entrevoir l'émergence d'univers (sémantiques) qui mettent en exergue des tensions, voire des " luttes axiologiques ${ }^{10}$ entre les acteurs de la consultation : l'État, les organisations et les citoyen(ne)s. La modélisation de ces investissements, sous la forme d'une structure ${ }^{11}$, offrira la possibilité d'expliciter la dynamique relationnelle à l'œuvre dans la consultation à travers certains leviers de la transformation agricole. Nous verrons un ensemble de valeurs " descriptives ${ }^{12}$ qui sont investies dans ces propositions, au sens où elles renvoient à des " registres de valeurs " tels que l'affectif, l'économique, le technique ou l'éthique ${ }^{13}$. Elles nous amèneront à aborder les modalités sémiotiques (ou valeurs «modales ») en termes de devoir et vouloir faire ${ }^{14}$.

Ainsi, à la lecture des propositions deux " grands " modèles émergent et s'opposent : le modèle de l'agriculture conventionnelle et le modèle des agricultures alternatives. Loin d'être des oppositions catégorielles ${ }^{15}$, l'approche sémiotique met l'accent sur les relations, mouvantes dans le temps et l'espace. Ces relations retiennent notre attention, car ce sont elles qui sont détentrices de valeurs socio-culturelles en l'occurrence. Selon A. J. Greimas :

" [C]'est la relation seule qui institue les "propriétés" [des objets du monde] : ces dernières, à leur tour, servent de déterminations pour les objets et les rendent [de fait] connaissables ${ }^{16}$.

La valorisation d'un modèle s'institue dans son opposition (sémantique) à un autre modèle. Ce principe définit une structure (sémiotique), où les relations, et non les objets (qu'ils soient réels ou conceptuels), sont vectrices de

9 Claude Zilberberg, La Structure tensive, Liège, Presses universitaires de Liège, 2012 [en ligne].

10 Olivier Turbide, Thomas Maxwell, «Les régimes de rationalité en environnement à travers le prisme des valeurs ", Argumentation et analyse du discours, 25, 2020 [en ligne].

11 Lopposition de deux termes (a minima). Joseph Courtés, Analyse sémiotique du discours. De l'énoncé à l'énonciation, Paris, Hachette supérieur, 1991, p. 152-160. Algirdas Julien Greimas, Sémantique structurale, Paris, PUF, 1986.

12 A. J. Greimas, J. Courtés, Sémiotique, dictionnaire raisonné de la théorie du langage, Paris, Hachette supérieur, 1993, p. 414-415.

13 Olivier Turbide, Thomas Maxwell, «Les régimes de rationalité en environnement à travers le prisme des valeurs ", Argumentation et analyse du discours, 25, 2020 [en ligne].

14 A. J. Greimas, J. Courtés, Sémiotique, dictionnaire raisonné de la théorie du langage, Paris, Hachette supérieur, 1993, p. 414-415.

15 C'est-à-dire qui n'admettent pas de moyen terme. Joseph Courtés, Analyse sémiotique du discours. De l'énoncé à l'énonciation, Paris, Hachette supérieur, 1991, p. 71.

16 A. J. Greimas, J. Courtés, Sémiotique, dictionnaire raisonné de la théorie du langage, Paris, Hachette supérieur, 1993, p. 360-366. 
sens. Elles régissent et construisent les représentations parce qu’on peut mettre les objets en relation de dépendance à d'autres objets (comparables bien sûr), mais aussi, et peut-être surtout, à d'autres valeurs (descriptives et modales) dont ils sont la manifestation. Par exemple, on ne peut juger de la qualité d'un produit que parce qu'on sait (selon son propre système de valeur, individuel et collectif notamment) ce qu'est un «bon " produit, et ce qui n'en est pas un ${ }^{17}$. Il y a nécessairement un processus d'opposition relationnelle qui se met en place, souvent implicite. Dans une perspective de changement global, climatique notamment, ce cadre d'analyse invite à appréhender les contours, voire les tensions existantes au sujet des représentations sur le milieu agricole français et sa transformation.

Ainsi fondé sur l'opposition relationnelle, nous tenterons de pointer et d'expliciter les faits signifiants, reflets des "manières " de penser des entités constituant le corpus : l'État, les organisations et les citoyen(ne)s. Les énoncés étudiés seront associés à un ensemble de valeurs qui nous conduiront à aborder la problématique de la confiance qui traverse depuis plusieurs décennies le milieu agricole.

De fait, si l'objectif d'un changement agricole vise à passer d'un état premier à un autre, il suppose la transformation de celui-ci, le modèle dominant actuel étant celui de l'agriculture dite « conventionnelle ». Pour appréhender celui-ci, en matière relationnelle, on doit l'opposer à un autre modèle, celui des agricultures qu'on pourra nommer " alternatives». On obtient la structure minimale suivante, qui oppose également ce qui définit chacun des termes ${ }^{18}$ :

Modèle dit de l' " agriculture conventionnelle »

- Industriel : monocultures, grandes surfaces agricoles, fortement mécanisées, non biologiques (dite conventionnelle), usage d'intrants, voire d'OGM ;

- centré « Capital », structure salariale ;

- échelle globalisée (mondiale).

versus

17 Cf. l'étude d'Aurélie Kessous et d'Isabelle Chalamon : " "Dis-moi ce que tu manges et je te dirai qui tu es!" Approche sémiotique des représentations mentales des marques alimentaires ", Management \& Avenir, n' 70, 2014/4, p. 33-54.

18 Ronan Le Velly, «Dynamiques des systèmes alimentaires alternatifs », in Pasquale Lubello et al., Systèmes agroalimentaires en transition, Paris, éd. Quæ, 2017 ; Sébastien Bainville, "Transition agroécologique, quand l'agronomie interroge l'économie ", in Pasquale Lubello, Systèmes agroalimentaires en transition, éd. Quæ, 2017 ; Alain Falque, Pasquale Lubello, Leila Temri, "Introduction », Systèmes agroalimentaires en transition, Versailles, éd. Quæ, 2017 ; Joël Aubin, Catherine Donnars, Markéta Supkova et Bruno Dorin, "Méthodes d'évaluation. Panorama critique ", in Catherine Esnouf, Pour une alimentation durable, Paris, éd. Quæ, 2011 ; Antoine Bernard De Raymond, Frédéric Goulet, «Introduction - Les transformations de l'agriculture intensive. Éléments pour une sociologie des grandes cultures ", Sociologie des grandes cultures, Paris, éd. Quæ, 2014. 


\section{Modèle dit des " agricultures alternatives"}

- Paysan : diversité des productions, surfaces plus réduites, faiblement mécanisées, biologiques ;

- centré « Humain ", structure familiale ;

- échelle localisée (territorialisée).

Quand le modèle de l'agriculture conventionnelle se définit, selon les propos de Ronan Le Velly, comme industriel, capitaliste et globalisé, le modèle alternatif est, quant à lui, déterminé par une agriculture biologique, un commerce équitable, des circuits courts et de proximité ${ }^{19}$. Sébastien Bainville décrit de la même façon ces deux modèles agricoles, invitant de la sorte à ce même type d'opposition relationnel. Il contraste l'agriculture capitaliste à l'agriculture paysanne. La première est extensive en travail salarié et intensive en capital. Elle tend vers la monoculture industrielle, utilisant de façon intensive les moyens mécaniques et les intrants. Elle est également grande consommatrice d'espaces fonciers. À l'inverse, la seconde peut pallier l'absence de moyens financiers par la mobilisation d'une main-d'œuvre familiale et la diversification des cultures. Elle permet de limiter les intrants, les moyens mécaniques et les surfaces cultivées ${ }^{20}$. Au-delà d'une simple opposition, on peut parler d'une structure relationnelle de confrontation, car les investissements sémantiques qui définissent ces modèles semblent particulièrement antagonistes, voire incompatibles. Ces modèles prennent la forme d'un " affrontement " (cognitif, voire somatique) qui laisse comprendre que le passage d'un modèle à l'autre requiert nécessairement une transformation radicale.

Cette visualisation sous-tend une vision dichotomique du paysage agricole, ou un modèle suppose le rejet, voire l'incompatibilité de l'un et l'autre. Toutefois, les propos issus du corpus montreront un panel de nuances. En outre, d'après Alain Falque, Pasquale Lubello et Leila Temri, la transition d'un modèle à l'autre se présente comme un " état-seuil », où le basculement de l'un à l'autre est « le résultat final d'un processus patient d'accumulation progressive d'un certain potentiel ${ }^{21}$. "Ces propos supposent des modèles intermédiaires que nous tenterons d'expliciter au regard du corpus, notamment en interrogeant le " comment ": Comment passer d'un état à un autre à partir des valeurs en jeu. Le corpus, en tant qu'échantillon représentatif de la consultation citoyenne sur la transformation de l'agriculture française, sera appréhendé ici comme un indicateur des positions actuelles en matière de rationalités diverses qui se croisent, se confrontent ou s'ajustent.

19 Ronan Le Velly, "Dynamiques des systèmes alimentaires alternatifs ", in Pasquale Lubello et al., Systèmes agroalimentaires en transition, Paris, éd. Quæ, 2017, p. 149-158.

20 Sébastien Bainville, "Transition agroécologique, quand l'agronomie interroge l'économie ", in Pasquale Lubello, Systèmes agroalimentaires en transition, éd. Quæ, 2017, p. 19-70.

21 Alain Falque, Pasquale Lubello, Leila Temri, "Introduction ", Systèmes agroalimentaires en transition, Versailles, éd. Quæ, 2017, p. 7-18. 


\section{Passer d'un modèle à un autre}

Les propositions faites par les "États généraux " (celles qui sont aussi les plus votées) sont au nombre de quatre :

(i) les lycées agricoles doivent être mieux outillés pour enseigner les nouveaux modes de production ;

(ii) il faut revoir l'absence de séparation entre le métier de conseil et de vente d'intrants. Cette proposition pointe la problématique du conflit d'intérêts;

(iii) les modes de production doivent davantage être accompagnés. Il est particulièrement pointé les aides financières de la PAC pour les modèles agricoles dits "durables » : l'agroécologie, le biologique;

(iv) il faut renforcer le manque de références techniques pour les entreprises agricoles durables. Encore une fois, il est pointé la problématique de l'enseignement, mais aussi de la formation continue tout au long de la vie de l'agriculteur ;

Pour résumer, ces propositions pointent :

- l'enseignement et la formation continue (on le retrouve dans les propositions 1 et 4 );

- un soutien des modèles agricoles dits "durables" à travers les aides financières, comme le soutien de la PAC aux projets durables, mais aussi par des soutiens techniques, moraux et/ou administratifs (propositions 3);

- les relations d'ordre social entre les acteurs de la filière agroalimentaire, notamment entre les statuts de vendeurs et de conseillers de produits phytosanitaires (proposition 2).

Ensuite, si l'on observe l'ensemble des propositions faites par les organisations et les citoyen(ne)s, les thématiques les plus abordées sont, d'une part, celles concernant le développement ou la transition vers une agriculture biologique. On compte une dizaine de propositions qui vont directement dans ce sens. Elles sont envisagées comme faisables par la mise au point ou la réorientation des aides financières (la Politique agricole commune [la PAC] est régulièrement citée), par la baisse de charges, ou encore par le développement d'une consommation collective (dans les écoles, les Ehpad, ou les entreprises, etc.). Elles incitent à se tourner ouvertement vers une production biologique. On compte également six propositions directement liées aux aides financières. Cela se manifeste par le biais d'une meilleure redistribution (encore une fois, la PAC revient régulièrement), ou par la gratuité du label bio par exemple. Dans une moindre mesure, l'accès au foncier est aussi une source de préoccupations. On compte au moins quatre propositions qui lui sont directement liées (avec, par exemple, un meilleur accès au foncier pour les personnes non issues du monde agricole, ou une baisse des pressions immobilières, notamment sur l'abord des villes). 
Les propositions que l'on retrouve également à plusieurs reprises, mais de façon moindre par rapport aux précédentes (c'est-à-dire qui sont explicitées de deux à trois fois dans notre corpus), sont liées à la prise en compte du réchauffement climatique. Il est souligné l'idée qu'on doit s'y adapter par des changements agricoles "significatifs". Ces propositions liées au climat sont en lien assez direct avec les précédentes puisqu'elles pointent l'usage néfaste des pesticides, entre autres. Parmi les propositions qu'on retrouve de deux à trois fois dans le corpus, remarquons celles qui sont liées à un besoin de procéder à un état de l'art coût/bénéfice de ces dernières décennies dominées par l'agriculture dite "conventionnelle» (débutée depuis les années 1960-70). Notons également le souci de valoriser les circuits courts et la place des petits producteurs. Ces propositions vont souvent de pair avec l'idée d'une production locale et de saison. Il y a également la volonté de préserver, ou plutôt de redonner la liberté de cultiver le patrimoine semencier. Cette proposition tend vers un encouragement à la valorisation de la biodiversité des cultures en lien avec leur territoire, et en opposition au principe des monocultures dont les semences sont associées aux brevets (et à un marché mondialisé). Enfin, nous relevons quatre autres propositions : deux pointent le rôle de l'enseignement, et deux prônent le remplacement pur et simple du modèle de l'agriculture intensive (soit conventionnelle).

Relevées une à une, ces propositions se recoupent, montrant la complexité d'une transformation de l'agriculture dans la société française. Le nombre assez conséquent de domaines concernés, et surtout de variables en jeu (environnementales, biologiques, sanitaires, éducatives, socio-économiques et sociétales ${ }^{22}$ ), dont un certain nombre sont pointées dans ce corpus, montre l'ampleur de la tâche. Cela met en évidence le besoin d'une loi systémique avec les difficultés que cela représente ${ }^{23}$. Mais si toutes ces propositions tendent vers une transformation de l'agriculture, certaines d'entre elles semblent toutefois plus ou moins nuancées quant à la radicalité d'un changement du modèle dominant.

À ce sujet, les quatre premières propositions, celles faites par les États généraux, n’investissent pas complètement le champ sémantique du modèle agricole alternatif défini précédemment. Elles s'inscrivent plutôt dans un ajustement du premier en prônant la "stabilité » des entités existantes. En effet, il semble qu'on cherche à redonner du sens aux structures et aux pratiques en

22 Joël Aubin, Catherine Donnars, Markéta Supkova et Bruno Dorin, « Méthodes d'évaluation. Panorama critique ", in Catherine Esnouf, Pour une alimentation durable, Paris, éd. Quæ, 2011, p. 205-232.

23 Selon Joël Aubin, Catherine Donnars, Markéta Supkova et Bruno Dorin, "le défi posé à l'évaluation [d'une alimentation durable] est [...] bien de prendre en compte cette complexité systémique sans la décomposer en différentes parties, ce qui lui ferait perdre ses caractéristiques. " Joël Aubin, Catherine Donnars, Markéta Supkova et Bruno Dorin, " Méthodes d'évaluation. Panorama critique ", in Catherine Esnouf, Pour une alimentation durable, Paris, éd. Quæ, 2011, p. 205-232. 
place, celles qui ont promu, et promeuvent encore le modèle conventionnel (la PAC, les centres de formation, par exemple). La programmation visée tend ainsi vers une progressivité de la transformation agricole, sans toutefois viser à aboutir complètement au modèle d'agricultures alternatives (comme défini ci-dessus). De plus, en nous appuyant sur la typologie des registres de valeurs de Nathalie Heinich ${ }^{24}$, on constate des investissements sémantiques de l'ordre de l'économique, du fonctionnel, et du technique. Les États généraux prônent particulièrement un accompagnement en matière de formation, de conseils techniques, et de soutien financier.

En revanche, les propositions les plus récurrentes des organisations et des citoyen(ne)s placent davantage en tête une transformation tournée, cette fois-ci, vers le modèle d'agricultures alternatives, où l'agriculture biologique est en tête. On l'observe par la volonté explicite de développer cette dernière, une dizaine de propositions va directement dans ce sens. L'idée d'une transformation, investissant le champ sémantique du deuxième modèle agricole, est aussi renforcée par les propositions sur les dérèglements climatiques, le besoin d'un état de l'art sur l'agriculture conventionnelle, ou celles pour le remplacement pur et simple du modèle de l'agriculture intensive. En outre, le modèle agricole conventionnel est à plusieurs reprises ouvertement pointé du doigt pour être remplacé, tout du moins réformé en profondeur. Avec les propositions des organisations et des citoyen(ne)s, on retrouve des investissements sémantiques liés aux registres de la technique, du fonctionnel ou de l'économique. Ils pointent, par exemple, de nouveaux secteurs d'activités créateurs d'emplois ${ }^{25}$. Mais ces investissements sémantiques revêtent également le registre de l'éthique, à travers un attachement au respect de l'environnement et à la santé des gens, ou celui de l'épistémique, avec la demande de réaliser un état de l'art, comme celui du civique, par le souci de propositions en lien avec l'intérêt général. Par certains aspects, ils engagent aussi le registre de l'affectif, par un attachement à une agriculture paysanne liée à des territoires, ou à la condition animale. On observe un besoin de transparence et d'une responsabilité des acteurs engagés dans le monde agricole à des fins d'intérêt général et environnementales. On s'inscrit ici dans une volonté de réinventer le modèle agricole actuel en invoquant des registres de valeurs variés et relevant autant d'états de choses (de l'intelligible) que d'états d'âme (du sensible).

Si les acteurs de la consultation s'entendent pour une transformation de l'agriculture à plus ou moins long terme, ils se différencient dans son incarnation, dans les valeurs soutenues : plus économiques, fonctionnelles et techniques d'un côté, également économiques, fonctionnelles et techniques de l'autre, mais

24 Olivier Turbide, Thomas Maxwell, "Les régimes de rationalité en environnement à travers le prisme des valeurs ", Argumentation et analyse du discours, 25, 2020 [en ligne].

25 Ces registres sont particulièrement en lien avec les propositions des organisations. 
aussi éthiques, épistémiques et affectives. Ainsi, l'expression commune d'un besoin de redonner du sens au modèle agricole de demain épouse des parcours différenciés. Quand l'un (les États généraux) s'inscrit dans une perspective de l'intelligible avec l'ajustement, la stabilité et la progressivité technique et économique, l'autre (les organisations et les citoyen(ne)s) invite à une rupture, au remplacement en convoquant des registres de valeurs de l'intelligible autant que du sensible. Ainsi, le premier point de vue est dans la nuance et envisage le modèle de l'agriculture conventionnelle comme "bon » ou, dans tous les cas comme " pas si mauvais ». Il est davantage à améliorer qu'à remplacer, tout n'est pas à jeter. Son positionnement suppose aussi que tout n'est pas à retenir dans le second modèle (celui des agricultures alternatives). Alors que le second point de vue, par ses investissements sémantiques et les valeurs qu'ils portent, représente le modèle actuel comme « mauvais » et donc à réinventer. En matière de valorisation des modèles, l'écart est beaucoup plus important qu'il n'y paraît, opposant ces deux groupes que sont les États généraux d'un côté, et les citoyen(ne)s et organisations de l'autre.

Ainsi, avec les propositions des États généraux, un troisième modèle se dessine. Il propose quelque chose qui ne serait plus de l'ordre du " conventionnel », mais qui ne relève pas non plus du modèle "alternatif ». En tant que nouvelle forme, il peut soit être envisagé comme le modèle d'une agriculture "non conventionnelle ", c'est-à-dire qui valoriserait une sorte de " correctioninflexion " de la trajectoire initiale du développement "standard ${ }^{26}$ pour se rapprocher du modèle alternatif. Mais il peut aussi être envisagé comme le modèle d'une agriculture "non alternative " centrée sur la valorisation des choses à conserver du premier modèle. En effet, si les propositions des États généraux se positionnent dans une observation plus fine des cultures, une adaptation des usages à des contextes locaux, ainsi qu'une adéquation des pratiques à la sensibilité de l'agriculteur ${ }^{27}$, elles s'inscrivent toutefois dans la continuité du modèle dominant, soit dans une transformation somme toute mineure. L'ambiguïté du positionnement des États généraux rend assez difficile la saisie des contours du modèle agricole de " demain " qu'ils veulent dessiner. À l'inverse, les propositions des organisations et des citoyen(ne)s montrent l'envie de s'orienter vers le modèle des agricultures alternatives prônant des cultures biologiques, des circuits courts, la consommation de produits de saison, ou encore par la prise en compte de l'urgence climatique. Une méfiance au premier modèle est prégnante notamment à partir des propositions des registres invoquant des valeurs éthiques, épistémiques ou affectives. Afin de faire sens, ces registres mêlent les dimensions du "sensible " et de l'" intelli-

26 Alain Falque, Pasquale Lubello Pasquale, Leila Temri, "Introduction ", Systèmes agroalimentaires en transition, Versailles, éd. Quæ, 2017, p. 7-18.

27 Antoine Bernard De Raymond, Frédéric Goulet, op. cit. 
gible ». Ensemble, elles se présentent comme les leviers d'une transformation. Rendre compte uniquement de la dimension "intelligible ", comme le font les États généraux, crée de fait un risque de désenchantement des citoyen(ne)s envers la loi alimentation.

Ces propos préliminaires doivent, bien sûr, être approfondis.

\section{Une crise de confiance qui perdure}

Cette brève étude des propositions semble être le signe manifeste d'une crise de confiance qui perdure, voire qui perdurera. À partir d'une première étude de cette consultation, nous avons pointé la mise en tension des propositions (notamment entre celles des États généraux et des organisations et des citoyen(ne)s). En effet, même s'il y a une convergence pour une transformation du modèle agricole français, les registres de valeurs ne sont pas en totale adéquation. Il en découle un autre point critique au niveau des valeurs modales. Les obligations de l'État, ce qu'il « doit » faire ne trouve pas un écho fort dans les attentes des citoyens, dans leur "vouloir » faire pour opérer la transformation. Autrefois associé au "progrès ", au " développement ", voire à $l^{\prime}$ « excellence $»^{28}$, le modèle conventionnel ne les incarne plus au profit d'autres modèles qui lui sont soit contraires (comme le modèle agricole dit " alternatif»), soit contradictoires (comme le modèle agricole « non conventionnel»). Mais dans leur perspective de progressivité, le positionnement discursif des États généraux n’opère pas cette bascule (sémantique en l'occurrence).

Dans un souci de santé, d'égalité, ou encore de respect environnemental, les registres éthiques ou épistémiques notamment, et évoqués précédemment, imposent un rythme en faveur d'une transformation importante et rapide du modèle agricole dominant vers celui des modèles alternatifs. Pointons le fait que les préoccupations citoyennes s'insèrent dans des vécus qui, par accumulation, accentuent et intensifient les ressentis et les attentes envers l'État ${ }^{29}$. Elles sont les manifestations d'une sensibilité qui engendre notamment une baisse de confiance entamée depuis plusieurs décennies dans la relation entre l'État, les producteurs et les consommateurs. Cette baisse de confiance est peut-être même plus acerbe aujourd'hui si le rapport entre le vouloir des citoyen(ne)s n'est pas plus en adéquation avec le devoir de l'État, via la loi alimentation. L'État semble être davantage tourné vers des " états de choses ", quand les instances citoyennes investissent également des " états d'âme " ${ }^{30}$, essentiel dans la prise de conscience et l'engagement actif du changement. À

\section{Ibid.}

29 On a observé qu'on retrouve la problématique de l'environnement (changement climatique, biodiversité) dans les propositions citoyennes et associatives et non dans celles formulées par l'État au sujet de la transformation de l'agriculture.

30 Claude Zilberberg, La Structure tensive, Liège, Presses universitaires de Liège, 2012 [en ligne]. 
l'instar des propos de Claude Zilberberg, il est important de ne pas négliger les " états d'âme ", car ils se présentent comme une constante régissant la première (les "états de choses »), qui n'en est finalement qu'une variable ${ }^{31}$. Un rapport de force apparaît entre la dimension intelligible de la transformation et sa dimension sensible.

Cependant, certaines des propositions des organisations et des citoyen(ne)s ont été actées dans la loi alimentation. Il faut attendre de voir comment elles seront appliquées et comment elles résoudront ou non les problématiques soulevées dans la consultation. Entre cette dernière et l'application de la loi, des effets de compositions, de décompositions, de pondérations, ou encore d'agencements ${ }^{32}$ résulteront, et qu'il faudra observer et étudier.

Pour terminer, notons que cette étude nécessite d'être approfondie à travers un élargissement du corpus, notamment en intégrant plus de propositions, en prenant en compte les commentaires écrits par les votants (autant ceux qui sont " pour » que ceux qui sont « contre»), mais aussi en l'inscrivant dans le temps.

Céline Cholet Laboratoire Médiations, Information, Communication, Arts, EA 4426 Université Bordeaux Montaigne celine.cholet@u-bordeaux-montaigne.fr

\begin{abstract}
Résumé
Depuis les années 2000, les enjeux sanitaires et environnementaux, ainsi que les préoccupations liées au changement climatique occupent une place importante dans la gestion de la politique agricole (à l'échelle française comme européenne) ${ }^{33}$. Ce constat nous conduit à nous intéresser aux États généraux de l'alimentation et particulièrement à la plateforme participative de la consultation citoyenne (ouverte du 20 juillet au 10 novembre 2017) ${ }^{34}$. Cette dernière nous paraît être un espace privilégié pour aborder le point de vue de la société civile, ses attentes, ses préoccupations, mais aussi, sa représentation du monde agricole à travers un ensemble de registres de valeurs en circulation. Ainsi, en prenant appui sur la consultation citoyenne, nous nous interrogerons sur les questions suivantes : quelles propositions le panel de citoyen(ne)s retient-il pour répondre à la question "Comment accompagner la transformation de notre agriculture " ? Le climat et la préservation de la biodiversité sont-ils présents, si oui, comment ? Le modèle agricole dominant (l'agriculture dite " conventionnelle ») est-il remis en cause? Si oui, comment? Au final, quelle(s) représentation(s) des modèles agricoles les investissements sémantiques que nous relèverons suggèrent-ils ? À partir d'une approche sémiotique, nous tenterons d'apporter des réponses à nos interrogations.
\end{abstract}

31 Ibid.

32 Nelson Goodman, Manières de faire des mondes, Paris, Gallimard, 2006.

33 Henri Nallet, Philippe Tillous-Borde, "Pour une nouvelle politique agricole ", Les nouveaux équilibres agroalimentaires mondiaux, Paris, Presses Universitaires de France, 2011, p. 119-125.

34 Site de la consultation citoyenne : egalimentation.gouv.fr. 


\title{
Mots-clés
}

Modèles agroalimentaires, consultation citoyenne, sémiotique, valeurs, représentations.

\begin{abstract}
Since the 2000s, health and environmental issues, but also the climate change have occupied an important place in the management of agricultural policy (at both the French and European levels). This observation leads us to take an interest in the Food General Assembly and particularly in the participatory platform for citizen (open from July 20 to November 10, 2017). The citizen consultation seems to be a privileged space for approaching the point of view of civil society, its expectations, its concerns, but also its representation of the agricultural world through a set of registers of values in circulation. Thus, based on the citizen consultation, we will question on: what proposals does the citizens" panel retain to answer the question "How to support the transformation of our agriculture"? Are the climate and the preservation of biodiversity present, if so, how? Is the dominant agricultural model (so-called "conventional" agriculture) being in question? and how? In the end, what are the representation(s) of agricultural models from semantic investments? From a semiotic approach, we will try to provide answers to our questions.
\end{abstract}

\section{Keywords}

Agrifood models, citizen consultation, semiotics, values, representations. 